Université กn
de Montréal

Serge Sultan, Université de Montréal

How to cite this article:

Lamore, K., Bourdeau, C., Alos, N., Bertout, L., Curnier, D., Drouin, S., Krajinovic, M., Laverdiere, C., Lippe, S., Marcil, V., Robaey, P., Rondeau, E., Sinnett, D., \& Sultan, S. (2020). Contributing Factors of Unmet Needs Among Young Adult Survivors of Childhood Acute Lymphoblastic Leukemia with Comorbidities. Journal of Adolescent and Young Adult Oncology. doi:10.1089/jayao.2020.0090

DOI: $\quad$ https://doi:10.1089/jayao.2020.0090

PMID: $\quad 32924727$ 


\title{
Contributing Factors of Unmet Needs Among Young Adult Survivors of Childhood Acute Lymphoblastic Leukemia with Comorbidities
}

\author{
Kristopher LAMORE, PhD,${ }^{1,2,3}$ Camille BOURDEAU, BSc, ${ }^{2}$ Nathalie ALOS, MD, ${ }^{4,5}$ \\ Laurence BERTOUT, MSc, ${ }^{1}$ Daniel CURNIER, PhD,${ }^{1,6}$ Simon DROUIN, PhD, ${ }^{1}$ \\ Maja KRAJINOVIC, PhD, ${ }^{4,5}$ Caroline LAVERDIÈRE, MD, ${ }^{4,5}$ Sarah LIPPÉ, PhD,,${ }^{2,4}$ \\ Valérie MARCIL, PhD,${ }^{4,7}$ Philippe ROBAEY, MD, PhD ${ }^{4,8,9,10}$ Émélie RONDEAU, MSc ${ }^{1}$ \\ Daniel SINNETT, PhD, ${ }^{4,5}$ Serge SULTAN, $\mathrm{PhD}^{2,4,5}$ \\ 1- Sainte-Justine University Health Center (SJUHC), Montréal, Québec, Canada \\ 2- Department of Psychology, Université de Montréal, Montréal, Québec, Canada \\ 3- Université de Paris, LPPS, Boulogne-Billancourt, France \\ 4- Sainte-Justine UHC Research Centre, Montréal, Québec, Canada \\ 5- Department of Pediatrics, Université de Montréal, Montréal, Québec, Canada \\ 6- Department of Kinesiology, Université de Montréal, Montréal, Québec, Canada \\ 7- Department of Nutrition, Université de Montréal, Montréal, Québec, Canada \\ 8- Department of Psychiatry, Université de Montréal, Montréal, Québec, Canada \\ 9- Department of Psychiatry, University of Ottawa, Ottawa, Ontario, Canada \\ 10- Children's Hospital of Eastern Ontario, Ottawa, Ontario, Canada
}

\section{Correspondence}

Kristopher Lamore, PhD, Division of Hematology-Oncology, Sainte-Justine UHC, 3175, Chemin de la Côte-Sainte-Catherine, Montréal, H3T1C5, Québec, Canada.

Email: kristopher.lamore@u-paris.fr

Running head: unmet needs of childhood leukemia survivors Keywords: childhood acute lymphoblastic leukemia; late adverse effects; morbidities; unmet needs; supportive care needs; survivorship 


\title{
Contributing Factors of Unmet Needs Among Young Adult Survivors of Childhood Acute Lymphoblastic Leukemia with Comorbidities
}

\begin{abstract}
Purpose: This study aimed to: (1) describe the domains and levels of unmet needs of young adult survivors of childhood acute lymphoblastic leukemia (cALL) with comorbidities, and (2) to explore the factors associated with higher levels of unmet needs. Unmet need was considered as supportive care needs not met.

Methods: The most vulnerable cALL survivors from the PETALE study cohort completed the Short-Form Survivor Unmet Needs Survey (SF-SUNS), the Brief Pain Inventory (BPI) and the $15 \mathrm{D}$ instrument of health-related quality of life. Demographic and clinical information, including comorbidities, were obtained from medical records or self-reporting. The participants' needs and contributing factors to their needs were evaluated using non-parametric tests.

Results: Of the 72 participants, nine (13\%) reported moderate/high levels of overall unmet needs. 'Worry about earning money' (56\%) and 'Dealing with feeling tired' (51\%) were the most frequent unmet needs (all levels combined). The factors associated significantly with any domain of unmet needs were: having a comorbidity, reporting altered functional health status, high ALL risk status, pain, age (<26 years) and having previously received psychological support.

Conclusion: A minority of young adult survivors of cALL with comorbidities interviewed reported moderate/high levels of unmet needs. However, financial concerns and emotional health and relationship are the two domains of greatest need. Survivors with altered health condition are most at risk of experiencing moderate/high levels of unmet needs. If confirmed in larger samples, interventions should target modifiable contributors of unmet needs such as physical health and comfort, fatigue, and emotional health.
\end{abstract}




\section{Introduction}

Childhood acute lymphoblastic leukemia (cALL) accounts for nearly one-third of childhood cancers, representing at least 50,000 new cases diagnosed per year worldwide. ${ }^{1,2}$ Currently, the 5year survival rate of these patients exceeds $90 \%$ in developed countries, where current innovative protocol-driven treatments are available to children. ${ }^{3-5}$ cALL is one of the most curable cancers. ${ }^{6}$ Hence, much current research has focused on late adverse effects resulting in comorbidities, and on survivors' quality of life, to prevent these complications.

Cohort studies of cALL survivors ${ }^{7,8}$ have ascertained many late adverse effects in this population due to treatment toxicity and radiation exposure., ${ }^{7,9}$ Survivors frequently have cardiac (e.g hypertension), metabolic (e.g. diabetes, hypertension), bone (e.g. osteonecrosis), neurocognitive (e.g. attention, memory) and psychosocial complications (e.g. anxiety, depression). ${ }^{7-18}$ Survivors' health-related quality of life can also be greatly impacted, as highlighted in recent systematic reviews. ${ }^{19,20}$

These complications may be partially preventable. Indeed, there is reason to believe that nutrition education interventions may favorably impact cardiometabolic comorbidities. ${ }^{21-24}$ Physical activity can lead to general improvement in the metabolism of patients and to alleviating the adverse effects of treatments, and improve patients' quality of life. ${ }^{25-28}$ Psychosocial interventions targeting insomnia, psychological distress and family functioning can also improve patients' quality of life. ${ }^{29-34}$ However, these interventions are not systematically tailored for this population, and are often not based on a systematic collection of patients' and survivors' needs.

To optimize the specificity of patient care and to achieve patient-centered care, ${ }^{35,36}$ patients should be interviewed or included in discussions for refining intervention strategies for their difficulties and needs. ${ }^{29,33,37}$ The assessment of unmet supportive care needs, also termed unmet needs, can help address the gap between survivors' concerns and the level of assistance provided, which is 
central to formulating a supportive care strategy. ${ }^{38,39}$ Supportive care is defined as a personcentered approach to 'the provision of the necessary services for those living with or affected by cancer to meet their informational, spiritual, emotional, social, or physical needs during diagnosis, treatment, or follow-up phases including issues of health promotion and prevention, survivorship, palliation and bereavement' ${ }^{40(\mathrm{p} .374)}$ Unmet needs have been classified into 11 primary domains: physical, psychosocial/emotional, family-related, social, interpersonal/intimacy, practical, daily living, spiritual/existential, health system/information, patient-clinician communication, and cognitive. ${ }^{40,41}$ Collecting information on these survivors' needs may be key to offering appropriate targeted services in clinics. ${ }^{38,42}$ Importantly, we still have very limited knowledge on the needs of the vulnerable population of cALL survivors. ${ }^{43,44}$

Thus, our objectives were: (1) to describe the domains and levels of unmet needs in cALL young adult survivors presenting cardiac, metabolic, neurocognitive, psychological or bone comorbidities, and (2) to explore whether these needs were explained by health status, demographics or clinical history.

\section{Methods}

This analysis was part of the PETALE study, a multidisciplinary research project conducted at Sainte-Justine University Health Center (SJUHC, Montreal, Canada). The PETALE study aimed to comprehensively characterize late adverse effects and to identify the associated predictive biomarkers of long-term treatment-related complications in cALL survivors. The study was divided into two phases of recruitment, with the second phase recalling survivors who presented extreme phenotypes (late effects) in the first screening phase. ${ }^{45}$ For the present study, we focused on participants included in Phase II, who are the most vulnerable survivors of the cohort (details 
provided below) and conducted a cross-sectional analysis of the survey performed on this subgroup of cALL survivors.

\section{Participants}

In Phase I, cALL survivors diagnosed between 1987 and 2010 before the age of 19 years, treated according to Dana-Farber Cancer Institute (DFCI)/ALL Consortium protocols 87-01 to 05-01, ${ }^{46} \geq 5$ years from diagnosis with no recurrence, who spoke French or English and were able to complete self-rated questionnaires were invited to take part in the study. Patients who received a hematopoietic stem cell transplant or who experienced recurrence were excluded. Participants eligible for study Phase II were cALL young adult survivors with extreme phenotypes in at least one of the following domains: cardiopulmonary, metabolic, bone, psychosocial, or neurocognitive. We only included survivors aged $\geq 19$ years. For example, if their measures of anxiety, depression or distress taken during Phase I exceeded the cut-points for clinical levels, they were invited to complete the Phase II investigations. Consequently, the Phase II sample is composed of the most vulnerable participants of the PETALE study. The full details on the study design and inclusion criteria for Phase II are available in Marcoux et al. ${ }^{45}$ and its Supplemental Table S1.

\section{Procedure}

Participants and their parents (if appropriate) were met between April 2014 and December 2016. The participants who agreed to take part in the study came to the hospital for a day of testing. The tests included complete biological measures, physical health examination and psychosocial questionnaires. The questionnaires were completed at the hospital or at home, with a mail-back envelope to be sent within 3 weeks. 
The Ethics Review Committee of SJUHC approved the study protocol and it was conducted in accordance with the Declaration of Helsinki. Written informed consent was obtained from all participants.

\section{Measures}

Demographic and clinical variables

Participant age, sex, ethnicity, marital status, family structure, educational background and professional status were self-reported. Time since diagnosis, overall clinical history including treatments, and information about whether they had received psychological support were collected from the patients' medical files.

\section{$\underline{\text { Comorbidities }}$}

For all Phase II participants, we also collected comorbidities clinically. The clinical director of the long-term follow-up clinic rated these as present or absent based on the medical files and clinical exams available at the assessment point using the Common Terminology Criteria for Adverse Events (CTCAE) version 4.03. ${ }^{47}$ No rating was available for psychological morbidity, as no systematic assessment of psychosocial distress was performed at the institution at the time of data collection. A morbidity in one domain was considered present if the domain required in-depth investigation or treatment. Examples of morbidities are: mineral density deficits or osteoporosis (bone), valvular abnormalities or ventricular arrhythmia (cardiac), attention or concentration deficits (neurocognitive), hyperlipidemia or type 2 diabetes (metabolic). This rating was made available for further analysis in the case report form. 


\section{$\underline{\text { Unmet needs }}$}

The participants' unmet needs were assessed using the Survivor Unmet Needs Survey short form (SF-SUNS). ${ }^{48}$ We adapted the questionnaire into French following standard translation procedures. ${ }^{49}$ The 30 -item questionnaire assesses four unmet need domains divided into four factors: (1) information ( 3 items; $\alpha=0.67$ ), (2) financial concerns ( 8 items; $\alpha=0.84$ ), (3) access and continuity of care (6 items; $\alpha=0.90$ ), and (4) emotional health and relationship (13 items; $\alpha=0.93$ ). Each domain is scored 0-4, with higher scores indicating high levels of unmet needs. Scores of 0 were classified as no unmet needs, $0-1$ as low unmet needs, 1-2 as low to moderate unmet needs, and 3-4 as high unmet needs. ${ }^{50}$

\section{$\underline{\text { Functional health status }}$}

The French version of the 15D instrument of health-related quality of life (http://www.15dinstrument.net/15d// $)^{51}$ was used to measure functional health status at study Phase I (1.25 \pm 0.60 years prior to Phase II) across 15 domains $(\alpha=0.82)$ : mobility, vision, hearing, breathing, sleeping, eating, speech, excretion, usual activities, mental function, discomfort and symptoms, depression, distress, vitality, and sexual activity. A single index score is obtained, ranging from 0 (being dead) to 1 (no problem in any dimension).

\section{$\underline{\text { Clinical pain }}$}

The French version of the Brief Pain Inventory (BPI) ${ }^{52,53}$ was used to measure both pain severity ( 4 items; $\alpha=0.87$ ) and pain interference ( 7 items; $\alpha=0.93$ ). Pain severity was measured at its worst, least, average and current level. The level of functional interference caused by pain was measured with items assessing general activity, walking, work, mood, sleep, enjoyment of life and relations 
with others. Scores of 1-4 were classified as mild pain, 4-7 as moderate pain and 7-10 as severe pain. $^{54}$

\section{Statistical analysis}

The Statistical Package for the Social Sciences (SPSS, version 25) was used to conduct statistical analyses. First, we conducted descriptive analyses on the different measures to confirm suitability for parametric tests (data normality and homogeneity when applicable). Then, for Objective 1, we computed means, standard deviations and frequencies for each factor and item of the SF-SUNS, and described the most frequent needs expressed. For Objective 2, non-parametric tests were performed. Mann-Whitney $U$ tests were used to explore whether unmet needs were linked to comorbidities, demographic and clinical characteristics. Considering possible multi-morbidity, we analyzed the links between unmet needs and the number of combined comorbidities with Spearman correlations. We also computed Spearman correlation tests to investigate the associations between unmet needs (SF-SUNS), clinical pain (BPI) and functional health status (15D).

\section{Results}

Participants characteristics

A total of 247 eligible cALL survivors were recruited in the PETALE Phase I study. From Phase II, 219 cALL survivors were eligible, among whom 72 young adults ( $\geq 19$ years) composed the present study sample (flow chart in Appendix Figure 1). The participants were aged 19-42 years (mean age, $27 \pm 6$ years), with a majority of self-reported Caucasian ethnicity (96\%). The mean time since diagnosis was $17 \pm 6$ years. Most participants had been treated with cranial radiotherapy (81\%; maximum dose was less than or equal to 19 Gy) and approximately one-quarter had received support from a mental health specialist. 
Most participants had at least one clinically significant comorbidity $(\mathrm{n}=56 ; 78 \%)$ according to the CTCAE rating: either one from among bone, metabolic or neurocognitive $(n=30 ; 54 \%)$ or a combination of bone, metabolic, neurocognitive or cardiac complications $(n=26 ; 46 \%)$. As summarized in Figure 1, the cardiac domain was always combined with another domain (metabolic, bone or neurocognitive). The most frequent morbidity domain alone was metabolic complications (56\%). On average, the participants had $1.30 \pm 1.00$ comorbidity. However, participants with neurocognitive complications were younger at diagnosis than participants without such complications $(6.31 \pm 4.25$ years vs. $10.67 \pm 4.92$ years, $U=274.00, p<.01)$. More specifically, patients who had radiotherapy and presented neurocognitive complications were younger at diagnosis than participants without such complications $(6.60 \pm 4.37$ years vs. $11.44 \pm 4.67$ years, $U=170.00, p<.01)$. No such statistical differences were observed for metabolic, bone or cardiac complications according to age at diagnosis (Appendix Table 1). Furthermore, the participants reported only mild levels of pain severity $(1.71 \pm 1.85$ on the $0-10$ scale) and pain interference (1.17 \pm 1.78 on the $0-10$ scale) as measured with the BPI, and very good overall functional health status $(0.92 \pm 0.08$ on the $0-1$ scale; $15 \mathrm{D}$ instrument $)$.

Table 1 lists the complete details of the participants' characteristics and present the differences between the participants included in PETALE study Phase I and Phase II.

[INSERT TABLE 1]

[INSERT FIGURE 1]

Description of unmet needs 
Most of the participants had no unmet needs (19\%) or low unmet need levels (68\%) (Table 2). The mean levels of unmet needs were also very low $(<0.60)$ for all domains. When present, unmet needs were more frequently reported in the emotional health and relationship domain. However, when investigating the responses for each item (Figure 2), we observed that $>40 \%$ of the participants rated five items as an unmet need, and $>20 \%$ of participants rated 15 items as an unmet need. Thus, some items appeared more representative of unmet needs in the present sample. Participants frequently reported being 'Worried about earning money' (item 4; 55.56\%) and expressed concerns about 'Paying household bills or other payments' (item 6; 43.06\%), highlighting financial concerns. Other needs were linked to emotions, such as 'Dealing with feeling tired' (item 24; $51.39 \%$ ), 'Dealing with feeling stressed' (item 25; 48.61\%) and 'Dealing with changes in how my body appears' (item 29; 47.22\%). Information, access and continuity of care were the domains with the fewest unmet needs for all items (reported by $<20 \%$ of the participants) (Figure 2).

\section{[INSERT TABLE 2]}

\section{[INSERT FIGURE 2]}

When exploring the associations among the domains of unmet needs, we found moderate-strong positive and significant correlations between all domains. The emotional health and relationship domain was strongly associated with both the financial concerns and information domains ( $\mathrm{r}=0.64$, $p<.01 ; \mathrm{r}=0.44, p<.01$, respectively) and was moderately associated with the access and continuity of care domain $(\mathrm{r}=0.33, p<.01)$. The access and continuity of care domain was also strongly associated with the financial concerns and information domains $(\mathrm{r}=0.47, p<.01 ; \mathrm{r}=0.43, p<.01$, 
respectively). We found a strong correlation between the financial concerns and information domains $(\mathrm{r}=0.40, p<.01)$.

\section{Contributing factors of unmet needs}

\section{$\underline{\text { Comorbidities }}$}

We explored the distribution of unmet needs across the domains of deteriorated health (Appendix Table 2). Participants with neurocognitive issues had higher levels of information $(p<.05)$ and emotional health and relationship unmet needs $(p<.05)$ than participants without neurocognitive late adverse effects. Participants with bone late adverse effects had higher levels of access and continuity of care $(p<.05)$ unmet needs than participants without bone complications. We found no difference for metabolic and cardiac comorbidities.

When examining the number of unmet needs according to the number comorbidities, we observed that more frequent comorbidities were accompanied by higher levels of unmet needs. This was particularly true for access and continuity of care $(\mathrm{r}=0.26, p<.05)$ (Figure 3$)$.

To control for multiple comorbidities, we removed participants presenting mixed profiles $(\mathrm{n}=26$,

Figure 1), and explored the level of unmet needs across the remaining three groups presenting only one comorbidity, i.e. metabolic $(n=15)$, bone $(n=10)$ or neurocognitive $(n=5)$. The results suggested that participants with metabolic complications had fewer unmet needs (all domains) than participants with bone or neurocognitive complications, but these apparent differences did not reach significance in this limited sample ( $p>.05)$ (Appendix Table 3).

\section{[INSERT FIGURE 3]}

$\underline{\text { Functional health status and clinical pain }}$ 
All domains of unmet needs were associated with functional health status and clinical pain (except for the financial concerns domain unrelated with clinical pain). Functional health status measured in Phase I was significantly and negatively associated with unmet needs (all domains). For example, worse functional health status was associated with more information needs and financial concerns $(\mathrm{r}=-0.32, p<.05 ; \mathrm{r}=-0.25, p<.05$, respectively). Unmet needs were also significantly and positively associated with pain interference (information, access and continuity of care, and emotional health and relationship domains) and pain severity (access and continuity of care, emotional health and relationship domains). Higher pain was associated with more frequent unmet needs. The complete results are available in Appendix Table 4.

\section{$\underline{\text { Demographic and clinical factors }}$}

We also found that unmet needs were associated with younger age, previous psychological support received, and higher ALL risk status. Financial concerns were more frequent among participants aged $<26$ years and those who had received psychological support ( $p<.05$ and $p<.01$, respectively). Participants who had received psychological support expressed more emotional health and relationship needs $(p<.05)$. Participants with high ALL risk status reported access and continuity

of care needs more frequently compared to those with standard risk $(p<.05)$. No significant association was found with sex, marital status, having children, time since diagnosis and radiotherapy received (Appendix Table 2).

\section{Discussion}

This study is the first to investigate the unmet supportive care needs of cALL young adult survivors and their contributing factors. The cALL survivors interviewed expressed few unmet needs. However, the most frequently expressed needs were related to the emotional health and 
relationship, and financial domains. The factors contributing to unmet needs, depending on the domain, were late adverse effect, altered functional health, pain, having received psychological support in the past and being younger than 26 years old. Healthcare professionals could use this information to quickly identify and assist those in need.

Twelve percent of our participants expressed moderate to high levels of unmet needs. This result is consistent with the literature, where the levels of unmet needs among survivors of hematologic cancer are generally low. ${ }^{55-58}$ A study conducted specifically on survivors of pediatric cancers, however, showed high levels of unmet needs. These needs were higher among leukemia cancer survivors compared to survivors of solid tumors. ${ }^{44}$ The differences found between our study and other studies can be linked to the country healthcare system and long-term care available. In Canada, universal healthcare exists. Our results are thus generalizable to countries with such healthcare system. However, even if our participants tended to express few unmet needs, more than $40 \%$ of them reported specific needs related to dealing with feeling stressed or tired, dealing with changed body and worrying about earning money and paying household bills or other payments. Indeed, psychological and information needs have been identified as the most frequent needs among adult cancer survivors, and physical and daily living concerns are the most frequent among adult hematological cancer survivors. ${ }^{59}$ Thus, cALL survivors seem to have similar unmet needs to other cancer survivors. Yet, among the unmet need domains identified in the literature, specific to cancer survivors ${ }^{60}$ or not, ${ }^{40,41}$ an important one has not been explored in our study, i.e. intimacy and sexuality. It appears particularly important to explore this domain among cALL survivors, as recent studies have reported that intimacy and sexuality are strongly impacted. ${ }^{61-63}$ This is even more obvious at an age when the child, adolescent or young adult is in the process of building their identity and exploring intimate relationships with others. ${ }^{64,65}$ In addition, childhood leukemia cancer survivors have fewer higher education and occupational achievements. ${ }^{66}$ Future qualitative 
studies may be particularly relevant for exploring a person's needs in these domains. Moreover, to our knowledge, no study explored unmet needs among other pediatric populations in a similar manner. Further studies should explore unmet needs in other oncologic and non-oncologic chronic childhood conditions to explore whether these needs can be condition- or age-related.

The participants of the present study were specifically selected based on health criteria. In this group, we found that the following factors related to their condition were associated with unmet needs: clinical comorbidities, functional health status and pain. Participants presenting several comorbidities expressed moderate/high needs for information and access and continuity of care. More specifically, the presence of neurocognitive morbidity was significantly associated with information and emotional health and relationship needs. Likewise, bone morbidities were associated with the need for access and continuity of care. A systematic review of adult cancer survivors found that comorbidities, symptoms and quality of life were associated with higher levels of unmet needs. ${ }^{59}$ In the present study, younger age was also associated with unmet needs. Participants younger than 26 years old reported more financial concerns than the older, highlighting an issue that may be specific to cALL survivors, as financial concerns are less prevalent in adult survivors of hematological cancers. ${ }^{55-58}$ This is probably the result of higher financial strain on pediatric cancer survivors resulting from their younger age and the responsibilities that accompany young adulthood.

We should acknowledge several limitations of this study. First, the limited sample size did not allow us to carry out multivariate analyses that would have allowed us to isolate the unique effects of the contributing factors. As Figure 1 illustrates, a detailed description of the participants' clinical context quickly yields small cell numbers. Second, $96 \%$ of our participants were Caucasians. Thus, the results are not generalizable to all ethnic groups. Third, although we based our analysis on a recognized questionnaire of unmet needs, our data potentially overlooked some aspects of cALL 
survivors' unmet needs (e.g. intimacy-sexuality). To ensure appropriate and targeted care, future research should continue exploring cALL survivors' needs in larger samples, using surveys specifically developed for the younger adult population. Researchers should also consider conducting further studies in more varied samples.

In conclusion, in a group of 72 vulnerable cALL survivors, the most frequent unmet needs were related to financial concerns and emotional aspects. The levels of unmet needs were related to the presence of clinical comorbidity, lower functional status, pain, as well as younger age and higher risk status. The results stress the complexity of distinguishing and studying different groups of participants based on their clinical profiles, as multi-morbidity is frequent. Future research should expand this line of research by improving profiling methods in larger samples and specifying the needs surveyed in childhood cancer survivors to better describe the needs of young adult survivors, as most tools have been developed for the much older adult populations.

\section{Acknowledgments}

We would like to thank all the survivors who participated in the study, and the clinical research staff.

\section{Author Disclosure Statement}

No competing financial interests exist.

\section{Funding Information}

This work was supported by the Institute of Cancer Research of the Canadian Institutes of Health Research, in collaboration with C17 Council, Canadian Cancer Society, Cancer Research Society, Garron Family Cancer Centre at the Hospital for Sick Children, Ontario Institute for Cancer 
Research, Fonds de Recherche du Québec-Santé Cancer Grant, and Pediatric Oncology Group of Ontario grant number TCF 118694. Editorial assistance in English was financed by the Laboratory of Psychopathology and Health Processes (LPPS), Université de Paris.

\section{References}

1. Pui C-H, Yang JJ, Bhakta N, Rodriguez-Galindo C. Global efforts toward the cure of childhood acute lymphoblastic leukemia. Lancet Child Adolesc Health. 2018;2(6):440-454.

2. Rodriguez-Galindo C, Friedrich P, Alcasabas P, et al. Toward the cure of all children with cancer through collaborative efforts: pediatric oncology as a global challenge. J Clin Oncol. 2015;33(27):3065-73.

3. Hunger S, Lu X, Devidas M, et al. Improved survival for children and adolescents with acute lymphoblastic leukemia between 1990 and 2005: a report from the children's oncology group. J Clin Oncol. 2012;30(14):1663-69.

4. Pieters R, Groot-Kruseman HD, Velden V, et al. Successful therapy reduction and intensification for childhood acute lymphoblastic leukemia based on minimal residual disease monitoring: study ALL10 from the Dutch Childhood Oncology Group. J Clin Oncol. 2016;34(22):2591-601.

5. Vora A, Goulden N, Mitchell C, et al. Augmented post-remission therapy for a minimal residual disease-defined high-risk subgroup of children and young people with clinical standard-risk and intermediate-risk acute lymphoblastic leukaemia (UKALL 2003): a randomised controlled trial. Lancet Oncol. 2014;15(8):809-18.

6. International Agency for Research on Cancer. Estimated number of deaths in 2018, worldwide, both sexes, all ages. GLOBOCAN 2018. Accessed on May 8, 2020 from: https://gco.iarc.fr/survival/survmark/visualizations/viz2/?cancer_site=\%22Colon $\% 22 \&$ countr 
$\mathrm{y}=\% 22 \mathrm{Australia} \% 22 \&$ agegroup $=\% 22 \mathrm{All} \% 22 \&$ gender $=\% 22 \mathrm{All} \% 22 \&$ interval $=\% 221 \% 22 \& \mathrm{su}$ rvival_year $=\% 221 \% 22 \&$ measures $=\% 5 \mathrm{~B} \% 22$ Incidence $+\% 28 \mathrm{ASR} \% 29 \% 22 \% 2 \mathrm{C} \% 22$ Mortality $+\% 28 \mathrm{ASR} \% 29 \% 22 \% 2 \mathrm{C} \% 22 \mathrm{Net}+$ Survival $\% 22 \% 5 \mathrm{D}$

7. Bhakta N, Liu Q, Ness KK, et al. The cumulative burden of surviving childhood cancer: an initial report from the St Jude Lifetime Cohort Study (SJLIFE). Lancet. 2017;390(10112):256982.

8. Mody R, Li S, Dover DC, et al. Twenty-five-year follow-up among survivors of childhood acute lymphoblastic leukemia: a report from the Childhood Cancer Survivor Study. Blood. 2008;111(12):5515-23.

9. Williams HE, Howell CR, Chemaitilly W, et al. Diabetes mellitus among adult survivors of childhood acute lymphoblastic leukemia: a report from the St. Jude Lifetime Cohort Study. Cancer. 2020;126(4):870-78.

10. Anestin AS, Lippé S, Robaey P, et al. Psychological risk in long-term survivors of childhood acute lymphoblastic leukemia and its association with functional health status. Pediatr Blood Cancer. 2018;65(11):e27356.

11. Brinkman TM, Li C, Vannatta K, et al. Behavioral, social, and emotional symptom comorbidities and profiles in adolescent survivors of childhood cancer: a report from the Childhood Cancer Survivor Study. J Clin Oncol. 2016;34(28):3417.

12. Boulet-Craig A, Robaey P, Barlaam F, et al. Visual short-term memory activation patterns in adult survivors of childhood acute lymphoblastic leukemia. Cancer. 2019;125(20):3639-48.

13. Levy E, Samoilenko M, Morel S, et al. Cardiometabolic risk factors in childhood, adolescent and young adult survivors of acute lymphoblastic leukemia. Sci Rep. 2017;7(1): 1-9. 
14. Liu W, Cheung YT, Brinkman TM, et al. Behavioral symptoms and psychiatric disorders in child and adolescent long-term survivors of childhood acute lymphoblastic leukemia treated with chemotherapy only. Psychooncology. 2018;27(6):1597-1607.

15. Mulrooney DA, Hyun G, Ness KK, et al. The changing burden of long-term health outcomes in survivors of childhood acute lymphoblastic leukaemia: a retrospective analysis of the St Jude Lifetime Cohort Study. Lancet Haematol. 2019;6(6):e306-e316.

16. Nazari S, Koupaei MT, Shafiee A, et al. Emotional/Behavioral problems in children with acute lymphoblastic leukemia: a case-control study. Int J Hematol Oncol Stem Cell Res. 2014;8(2):120.

17. Petrykey K, Lipp S, Robaey P, et al. Influence of genetic factors on long-term treatment related neurocognitive complications, and on anxiety and depression in survivors of childhood acute lymphoblastic leukemia: The Petale study. PLoS One. 2019;14(6):e0217314.

18. Phillips NS, Howell CR, Lanctot JQ, et al. Physical fitness and neurocognitive outcomes in adult survivors of childhood acute lymphoblastic leukemia: a report from the St. Jude Lifetime Cohort. Cancer. 2020;126(3):640-48.

19. Fardell JE, Vetsch J, Trahair T, et al. Health-related quality of life of children on treatment for acute lymphoblastic leukemia: A systematic review. Pediatr Blood Cancer. 2017;64(9):e26489.

20. Vetsch J, Wakefield CE, Robertson EG, et al. Health-related quality of life of survivors of childhood acute lymphoblastic leukemia: a systematic review. Qual Life Res. 2018;27(6):14311443.

21. Hill R, Hamby T, Bashore L, et al. Early nutrition intervention attenuates weight gain for pediatric acute lymphoblastic leukemia patients in maintenance therapy. J Pediatr Hematol Oncol. 2018;40(2):104-10. 
22. Huang JS, Dillon L, Terrones L, et al. Fit4Life: a weight loss intervention for children who have survived childhood leukemia. Pediatr Blood Cancer. 2014;61(5):894-900.

23. Li R, Donnella H, Knouse $\mathrm{P}$, et al. A randomized nutrition counseling intervention in pediatric leukemia patients receiving steroids results in reduced caloric intake. Pediatr Blood Cancer. 2017;64(2):374-80.

24. Touyz L, Cohen J, Wakefield C, et al. Design and rationale for a parent-led intervention to increase fruit and vegetable intake in young childhood cancer survivors (Reboot): protocol for a pilot study. JMIR Res Protoc. 2018;7(5):e129.

25. Braam KI, van der Torr P, Takken T, et al. Physical exercise training interventions for children and young adults during and after treatment for childhood cancer. Cochrane Database Syst Rev. 2016;(3):CD008796.

26. Fiuza-Luces C, Padilla JR, Soares-Miranda L, et al. Exercise intervention in pediatric patients with solid tumors: the physical activity in pediatric cancer trial. Med Sci Sports Exerc. 2017;49(2):223-30.

27. Pedersen BK, Saltin B. Evidence for prescribing exercise as therapy in chronic disease. Scand J Med Sci Sports. 2006;16(Suppl 1):3-63.

28. Thorsteinsson T, Larsen HB, Schmiegelow K, et al. Cardiorespiratory fitness and physical function in children with cancer from diagnosis throughout treatment. BMJ Open Sport Exerc Med. 2017;3(1):e000179.

29. Barnett M, McDonnell G, DeRosa A, et al. Psychosocial outcomes and interventions among cancer survivors diagnosed during adolescence and young adulthood (AYA): a systematic review. J Cancer Surviv. 2016;10(5):814-31. 
30. Johnson JA, Rash JA, Campbell TS, et al. A systematic review and meta- analysis of randomized controlled trials of cognitive behavior therapy for insomnia (CBT-I) in cancer survivors. Sleep Med Rev. 2016;27:20-8.

31. Kazak AE, Alderfer MA, Streisand R, et al. Treatment of posttraumatic stress symptoms in adolescent survivors of childhood cancer and their families: a randomized clinical trial. J Fam Psychol. 2004;18(3):493-504.

32. McDonnell GA, Salley CG, Barnett M, et al. Anxiety Among Adolescent Survivors of Pediatric Cancer. J Adolesc Health. 2017;61(4):409-23.

33. Ogez D, Péloquin K, Bertout L, et al. Psychosocial intervention programs for parents of children with cancer: a systematic review and critical comparison of programs' models and development. J Clin Psychol Med Settings. 2019;26(4):550-74.

34. Zhou ES, Vrooman LM, Manley PE, et al. Adapted delivery of cognitive-behavioral treatment for insomnia in adolescent and young adult cancer survivors: a pilot study. Behav Sleep Med. 2017;15(4):288-301.

35. Australian Commission on Safety and Quality in Health Care. (2011). Patient-Centred Care: Improving quality and safety by focusing care on patients and consumers. Australian Commission on Saftey and Quality in Health Care ACSQHC, Sydney. Accessed on May 8, 2020 from: https://www.safetyandquality.gov.au/sites/default/files/migrated/PCC_Paper_August.pdf

36. Hanna A. Patient-centred care. Ontario Med Review. 2010;1:27.

37. Ogez D, Bourque CJ, Péloquin K, et al. Definition and improvement of the concept and tools of a psychosocial intervention program for parents in pediatric oncology: a mixed-methods feasibility study conducted with parents and healthcare professionals. Pilot Feasibility Stud. 2019;5:20. 
38. Campbell HS, Sanson-Fisher R, Turner D, et al. Psychometric properties of cancer survivors' unmet needs survey. Support Care Cancer. 2011;19(2):221-30.

39. Sanson-Fisher R, Girgis A, Boyes A, et al. The unmet supportive care needs of patients with cancer. Cancer. 2000;88(1):225-36.

40. Hui D. Definition of supportive care: does the semantic matter? Curr Opin Oncol. 2014;26(4):372-9.

41. Paterson C, Robertson A, Smith A, Nabi G. Identifying the unmet supportive care needs of men living with and beyond prostate cancer: a systematic review. Eur J Oncol Nurs. 2015;19:40518.

42. Wen KY, Gustafson D. Needs assessment for cancer patients and their families. Health Qual Life Outcomes. 2004;2:11.

43. Barata A, Wood WA, Choi SW, Jim HS. Unmet needs for psychosocial care in hematologic malignancies and hematopoietic cell transplant. Curr hematol Malig Rep. 2016;11(4):280-87.

44. Cox CL, Zhu L, Ojha RP, et al. The unmet emotional, care/support, and informational needs of adult survivors of pediatric malignancies. J Cancer Surviv. 2016;10(4):743-58.

45. Marcoux S, Drouin S, Laverdière C, et al. The PETALE study: late adverse effects and biomarkers in childhood acute lymphoblastic leukemia survivors. Pediatr Blood Cancer. 2017;64(6):e26361.

46. Silverman LB,Stevenson KE, O'Brien JE, et al. Long-term results of Dana-Farber Cancer Institute ALL Consortium protocols for children with newly diagnosed acute lymphoblastic leukemia (1985-2000). Leukemia. 2010 ;24(2):320-34.

47. National Cancer Institute. Common Terminology Criteria for Adverse Events (CTCAE). $\begin{array}{lllll}\text { Accessed } & \text { on } & \text { May } & \text { 8, } & 2020\end{array}$ https://ctep.cancer.gov/protocolDevelopment/electronic_applications/ctc.htm 
48. Campbell HS, Hall E, Sanson-Fisher RW, et al. Development and validation of the Short-Form Survivor Unmet Needs Survey (SF-SUNS). Support Care Cancer. 2014;22(4):1071-9.

49. Vallerand RJ. Toward a methodology for the transcultural validation of psychological questionnaires: Implications for Studies in the French language. Can Psychol. 1989;30:662-80.

50. Filsinger S, Bukhalter R, Campbell S. Survivor Unmet Needs Survey. User Guide: Codebook, Analysis, etc. Accessed on May 8, 2020 from: https://topslide.net/document/survivor-unmetneeds-survey-user-guide-codebook-analysis-etc-prepared-by-stephanie-filsinger-msc-robinburkhalter-mmath-sharon-campbell-phd

51. Sintonen H. The 15D instrument of health-related quality of life: properties and applications. Ann Med. 2001;33(5):328-36.

52. Cleeland CS. Pain assessment in cancer. In: Osoba D (Ed). Effect of cancer on quality of life. Boca Raton: CRC Press; 1991. pp. 293-305.

53. Poundja J, Fikretoglu D, Guay S, Brunet A. Validation of the French version of the brief pain inventory in Canadian veterans suffering from traumatic stress. J Pain Symptom Manage. 2007.33(6):720-6.

54. Woo A, Lechner B, Fu T, et al. Cut points for mild, moderate, and severe pain among cancer and non-cancer patients: a literature review. Ann Palliat Med. 2015;4(4):176-83.

55. Hall A, D'Este C, Tzelepis F, et al. Factors associated with haematological cancer survivors experiencing a high level of unmet need across multiple items of supportive care: a crosssectional survey study. Support Care Cancer. 2014;22(11):2899-909.

56. Hall AE, Sanson-Fisher RW, Lynagh MC, et al. What do haematological cancer survivors want help with? A cross-sectional investigation of unmet supportive care needs. BMC Res Notes. $2015 ; 8: 221$. 
57. Oberoi DV, White VM, Seymour JF, et al. Distress and unmet needs during treatment and quality of life in early cancer survivorship: a longitudinal study of haematological cancer patients. Eur J Haematol. 2017;99(5):423-30.

58. Oberoi D, White VM, Seymour JF, et al. The influence of unmet supportive care needs on anxiety and depression during cancer treatment and beyond: A longitudinal study of survivors of haematological cancers. Support Care Cancer. 2017;25(11):3447-56.

59. Miroševič Š, Prins JB, Selič P, et al. Prevalence and factors associated with unmet needs in posttreatment cancer survivors: a systematic review. Eur J Cancer Care. 2019;28(3):e13060.

60. Harrison JD, Young JM, Price MA, et al. What are the unmet supportive care needs of people with cancer? A systematic review. Support Care Cancer. 2009;17(8):1117-28.

61. Greaves P, Sarker S, Chowdhury K, et al. Fertility and sexual function in long-term survivors of haematological malignancy: using patient-reported outcome measures to assess a neglected area of need in the late effects clinic. Br J Haematol. 2014;164(4):526-35.

62. Haavisto A, Henriksson M, Heikkinen R, et al. Sexual function in male long-term survivors of childhood acute lymphoblastic leukemia. Cancer. 2016;122(14):2268-76.

63. Nahata L, Morgan TL, Lipak KG, et al. Romantic Relationships and Physical Intimacy Among Survivors of Childhood Cancer. J Adolesc Young Adult Oncol. Forthcoming. https://doi.org/10.1089/jayao.2019.0114

64. Erikson, E. (1968). Identity: youth and crisis. New York: Norton.

65. Marcia JE. Identity six years later: a follow-up study. J Youth Adolesc. 1976;5:145-60.

66. Dumas A, Berger C, Auquier P, et al. Educational and occupational outcomes of childhood cancer survivors 30 years after diagnosis: a French cohort study. $\mathrm{Br}$ J Cancer. 2016;114(9):1060-68. 
Table 1. PETALE study Phase I and II $(\mathrm{n}=247$ and 72) participants' demographic and clinical characteristics.

\begin{tabular}{|c|c|c|c|c|c|}
\hline & \multicolumn{2}{|c|}{$\begin{array}{c}\text { Participants included } \\
\text { in study Phase II } \\
(\mathbf{n}=72)\end{array}$} & \multicolumn{2}{|c|}{$\begin{array}{c}\text { Participants included } \\
\text { in study Phase } I(n= \\
247)\end{array}$} & \multirow[t]{2}{*}{$p$} \\
\hline & $\mathbf{n}$ & $\%$ & $\mathbf{n}$ & $\%$ & \\
\hline \multicolumn{6}{|l|}{ Demographic characteristics } \\
\hline Sex & & & & & .613 \\
\hline Male & 38 & 52.78 & 122 & 49.39 & \\
\hline Female & 34 & 47.22 & 125 & 50.61 & \\
\hline Age (mean in years, $S D)$ & $26.79(5.89)$ & - & $21.65(6.34)$ & - & $<.001$ \\
\hline Marital status & & & & & $<.001$ \\
\hline Single & 38 & 52.78 & 187 & 75.71 & \\
\hline Married & 8 & 11.11 & 9 & 3.64 & \\
\hline Common law & 25 & 34.72 & 47 & 19.03 & \\
\hline Divorced & 0 & 0 & 4 & 1.62 & \\
\hline Missing data & 1 & 1.39 & 0 & 0 & \\
\hline Ethnicity & & & & & .737 \\
\hline Caucasian & 69 & 95.83 & 238 & 96.36 & \\
\hline Other & 3 & 4.17 & 9 & 3.63 & \\
\hline Occupation & & & & & $<.001$ \\
\hline Working full-time & 45 & 62.50 & 91 & 36.84 & \\
\hline Working part-time & 19 & 26.39 & 62 & 25.10 & \\
\hline Unpaid work or unemployed & 8 & 11.11 & 93 & 37.65 & \\
\hline Missing data & 0 & 0 & 1 & 0.41 & \\
\hline Number of children & & & & & .078 \\
\hline 0 & 53 & 73.61 & 214 & 86.64 & \\
\hline 1 & 7 & 9.72 & 17 & 6.88 & \\
\hline 2 & 9 & 12.50 & 12 & 4.86 & \\
\hline 3 or more & 3 & 4.17 & 4 & 1.62 & \\
\hline \multicolumn{6}{|l|}{ Clinical characteristics } \\
\hline $\begin{array}{l}\text { Time since diagnosis (mean } \\
\text { in years, } S D \text { ) }\end{array}$ & $16.58(5.96)$ & - & $15.54(5.20)$ & - & .180 \\
\hline DFCI protocol & & & & & .116 \\
\hline $87-01$ & 8 & 11.11 & 21 & 8.50 & \\
\hline $91-01$ & 18 & 25.00 & 46 & 18.62 & \\
\hline $95-01$ & 19 & 26.39 & 73 & 29.55 & \\
\hline $2000-01$ & 13 & 18.05 & 76 & 30.77 & \\
\hline $2005-01$ & 10 & 13.89 & 27 & 10.93 & \\
\hline Other & 4 & 5.55 & 4 & 1.62 & \\
\hline Radiotherapy & & & & & $<.05$ \\
\hline Yes & 58 & 80.55 & 147 & 59.51 & \\
\hline No & 14 & 19.45 & 100 & 40.49 & \\
\hline ALL risk status & & & & & $<.05$ \\
\hline High risk & 52 & 72.22 & 118 & 47.77 & \\
\hline Standard risk & 19 & 26.39 & 128 & 51.82 & \\
\hline Unclassified & 1 & 1.39 & 1 & 0.41 & \\
\hline
\end{tabular}




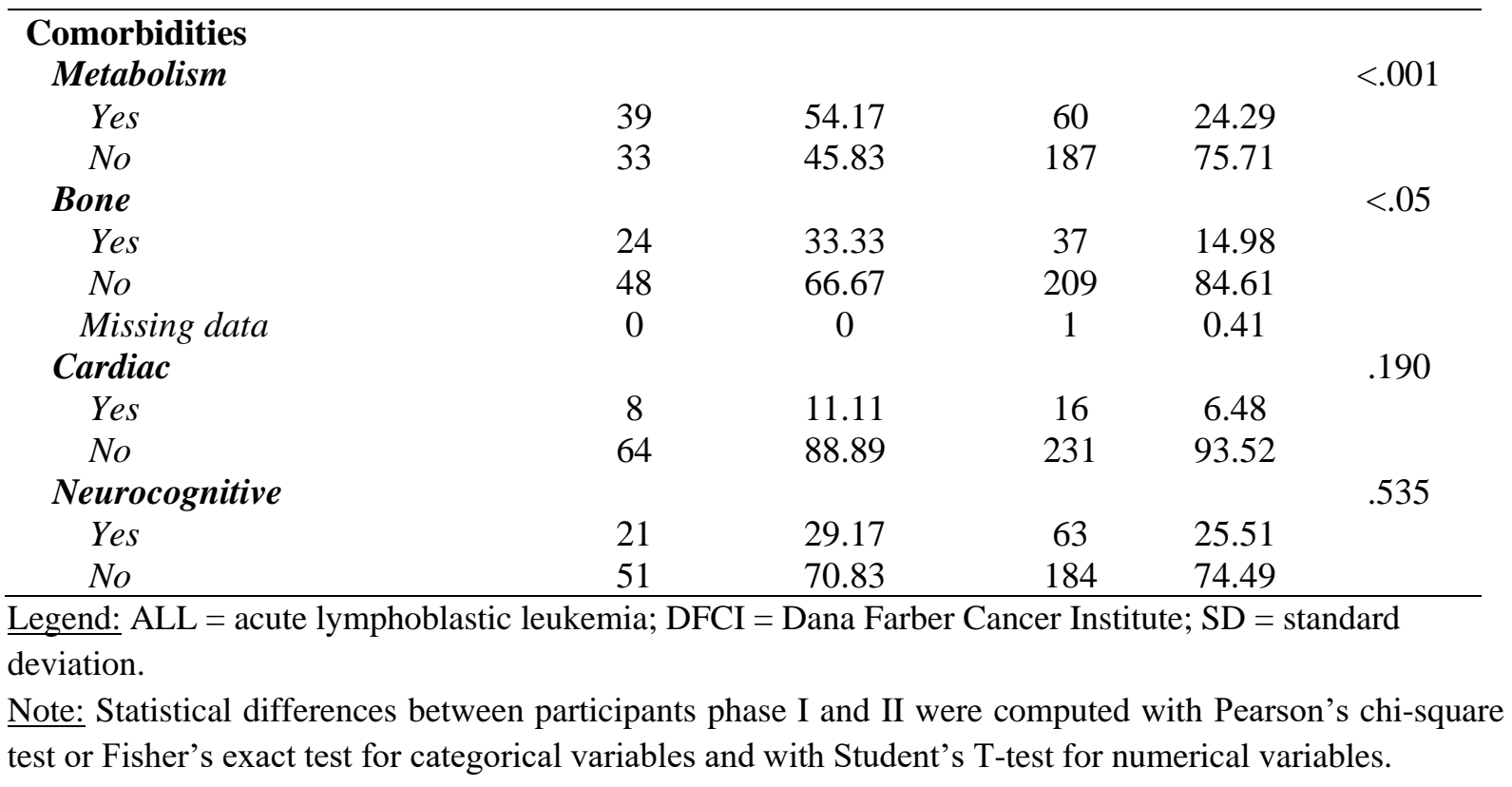


Table 2. Levels of unmet needs per domain on the SF-SUNS ( $n=72$ cALL survivors from the PETALE study).

\begin{tabular}{lccccc}
\hline Level of unmet need (up to 4) & Information & $\begin{array}{c}\text { Financial } \\
\text { concerns }\end{array}$ & $\begin{array}{c}\text { Access and } \\
\text { continuity of } \\
\text { care }\end{array}$ & $\begin{array}{c}\text { Emotional } \\
\text { health and } \\
\text { relationship }\end{array}$ & $\begin{array}{c}\text { Total } \\
\text { score }\end{array}$ \\
\cline { 2 - 6 } & $\mathbf{n ( \% )}$ & $\mathbf{n}(\boldsymbol{\%})$ & $\mathbf{n}(\boldsymbol{\%})$ & $\mathbf{n}(\boldsymbol{\%})$ & $\mathbf{n}(\boldsymbol{\%})$ \\
\hline No unmet need (0) & $53(74 \%)$ & $23(32 \%)$ & $47(65 \%)$ & $21(29 \%)$ & $14(19 \%)$ \\
Low unmet need (0 to 1) & $14(19 \%)$ & $40(56 \%)$ & $18(25 \%)$ & $30(42 \%)$ & $49(68 \%)$ \\
Low to moderate unmet need (1 to 3) & $5(7 \%)$ & $8(11 \%)$ & $6(8 \%)$ & $21(29 \%)$ & $9(13 \%)$ \\
High unmet need (3 to 4) & 0 & $1(1 \%)$ & $1(1 \%)$ & 0 & 0 \\
\hline Mean level of unmet need (SD) & $0.22(0.49)$ & $0.44(0.59)$ & $0.28(0.58)$ & $0.60(0.71)$ & $0.45(0.52)$ \\
Range & $0-2.67$ & $0-3$ & $0-3.17$ & $0-2.69$ & $0-2.3$ \\
\hline
\end{tabular}

Legend: $\mathrm{SD}=$ standard deviation. 
Figure 1. Co-occurrences of clinically rated comorbidities in adults from PETALE Phase II study ( $\mathrm{n}=56$ cALL survivors from the PETALE study).

Figure 2. Levels of unmet needs per item on the SF-SUNS ( $n=72$ cALL survivors from the PETALE study).

Items labels' (Campbell et al., 2014) : Item \#1: Finding information about complementary or alternative therapies; item \#2: Dealing with fears about cancer spreading; item \#3: Dealing with worry about whether the treatment has worked; item \#4: Worry about earning money; item \#5: Having to take a pension or disability allowance; item \#6: Paying household bills or other payments; item \#7: Finding what type of financial assistance is available and how to obtain it; item \#8: Finding car parking that I can afford at the hospital or clinic; item \#9: Understanding what is covered by my medical insurance or benefits; item \#10: Knowing how much time I would need away from work; item \#11: Doing work around the house (cooking, cleaning, home repairs etc.); item \#12: Having access to cancer services close to my home; item \#13: Getting appointments with specialists quickly enough (oncologist, surgeon etc.); item \#14: Getting test results quickly enough; item \#15: Having access to care from other health specialists (eg dieticians, physiotherapists, occupational therapists); item \#16: Making sure I had enough time to ask my doctor or nurse questions; item \#17: Getting the health care team to attend promptly to my physical needs; item \#18: Telling others how I was feeling emotionally; item \#19: Finding someone to talk to who understands and has been through a similar experience; item \#20: Dealing with people who expect me to be "back to normal"; item \#21: Dealing with people accepting that having cancer has changed me as a person; item \#22: Dealing with reduced support from others when treatment has ended; item \#23: Dealing with feeling depressed; item \#24: Dealing with feeling tired; item \#25: Dealing with feeling stressed; item \#26: Dealing with feeling lonely; item \#27: Dealing with not being able to feel 'normal'; item \#28: Trying to stay positive; item \#29: Coping with having a bad memory or lack of focus; item \#30: Dealing with changes in how my body appears.

Figure 3. Mean levels of unmet needs according to the number of comorbidities ( $\mathrm{n}=72 \mathrm{cALL}$ survivors from the PETALE study).

Legend: SF-SUNS $=$ The Survivor Unmet Needs Survey short form.

Note: Among all the participants, 16 participants had no comorbidity, 30 had one among metabolic, bone or neurocognitive, 26 had two to four comorbidities (error bars represent the standard deviation of the data set).

Appendix Figure 1. Flowchart of participants.

Legend: $\mathrm{cALL}=$ childhood acute lymphoblastic leukemia. 


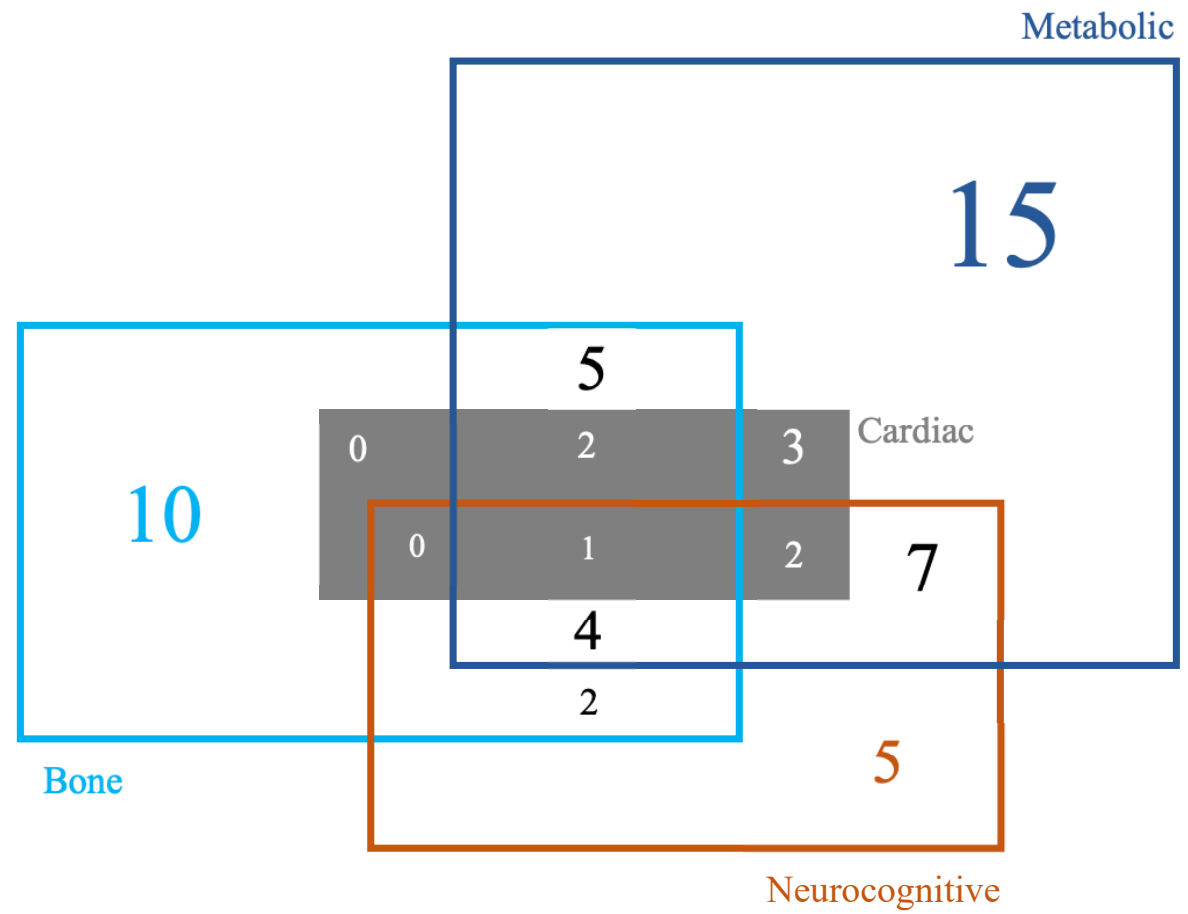




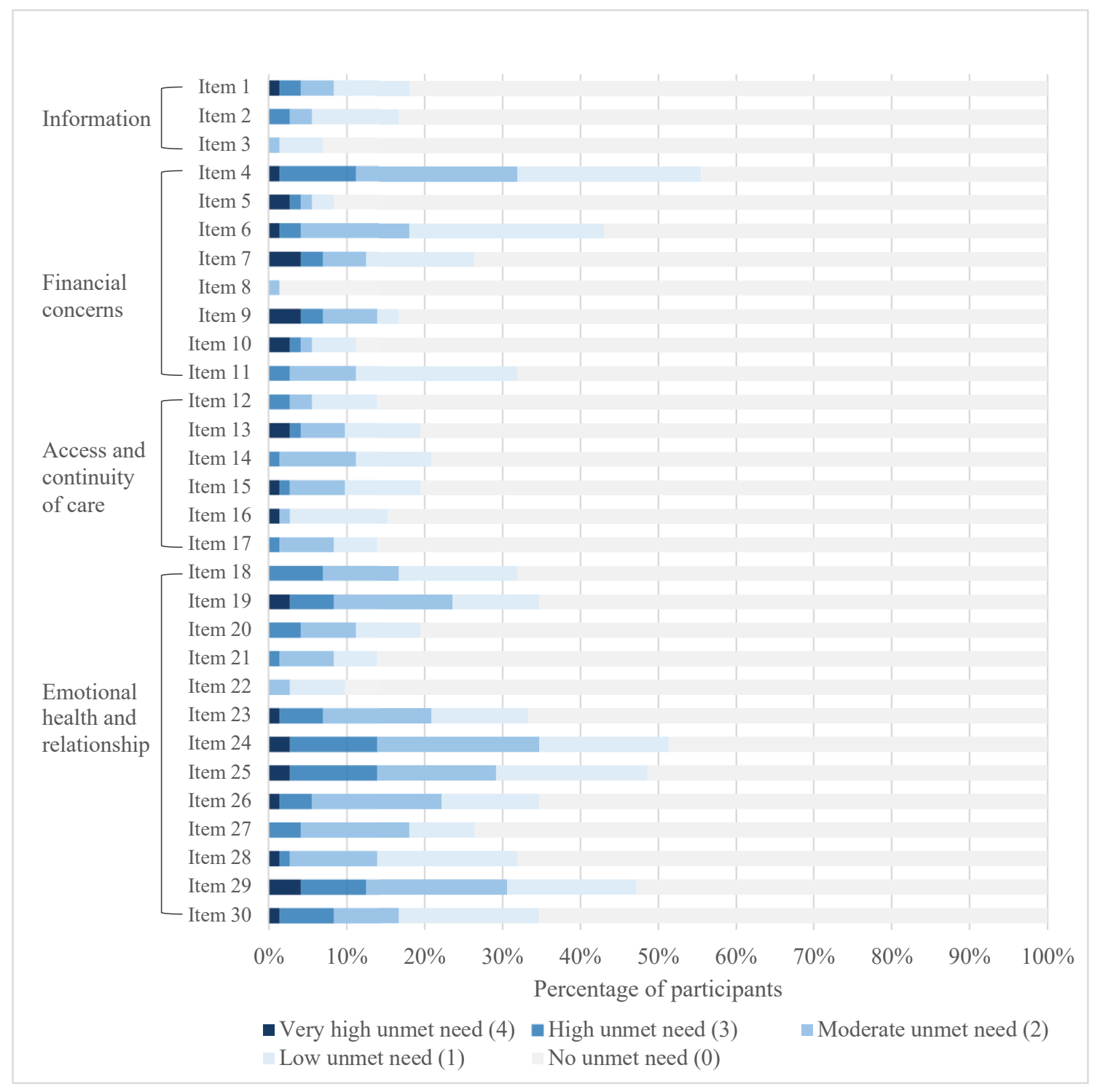




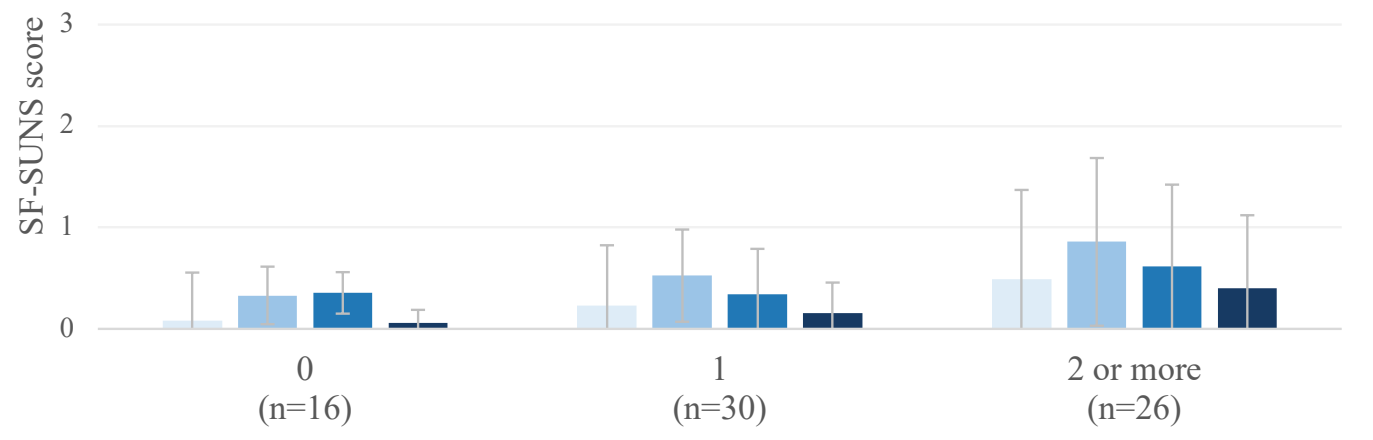

Number of comorbidities

Access and continuity of care Emotional health and relationship

- Financial concerns a Information 


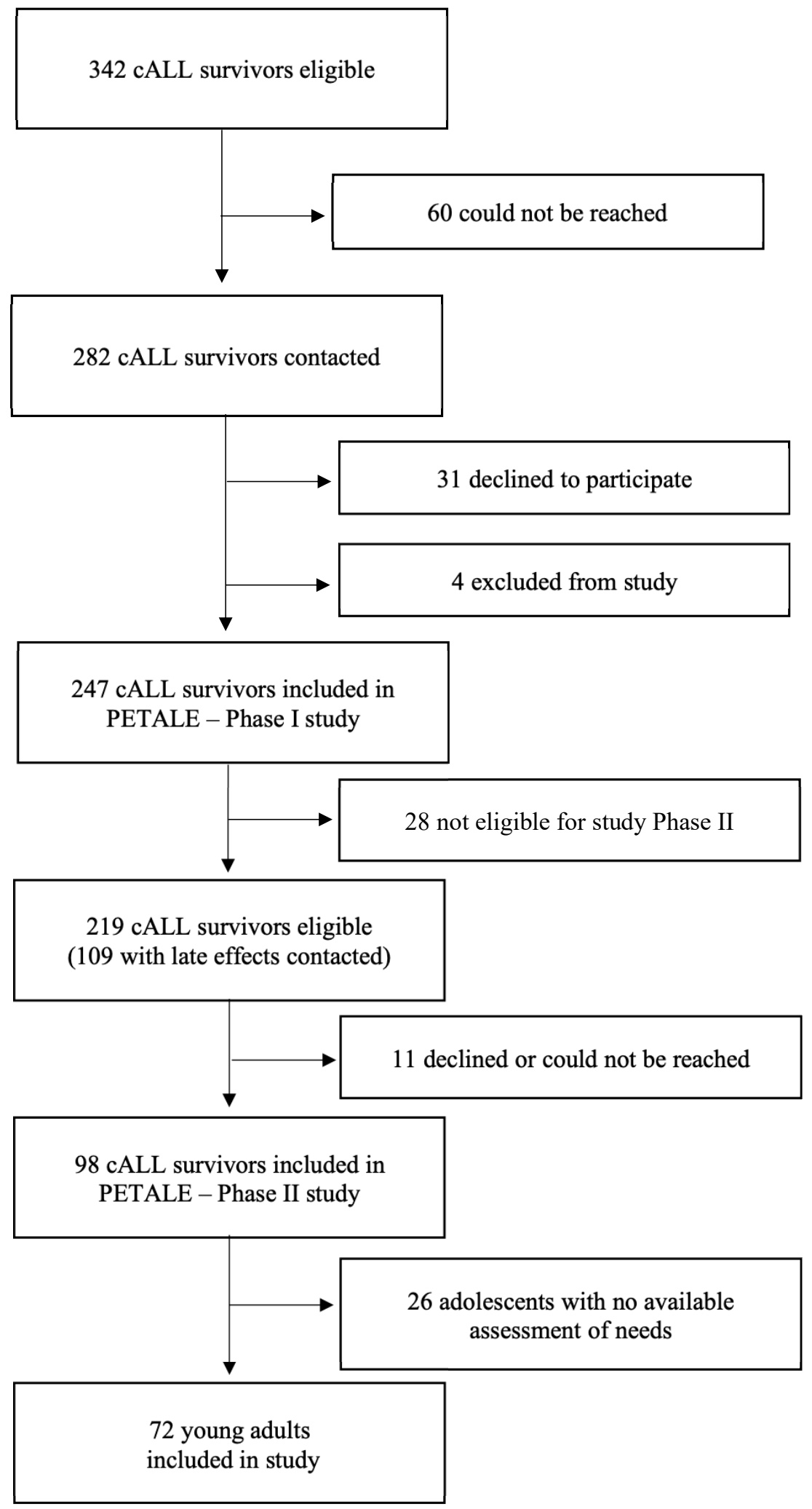


Appendix Table 1. Mean age at diagnosis according to the presence or absence of late adverse effects $(n=72)$.

\begin{tabular}{llcc}
\hline \multirow{2}{*}{ Late adverse effects } & \multicolumn{3}{c}{ Age at diagnosis (mean - SD) } \\
\cline { 2 - 4 } Metabolic complication & \multicolumn{1}{c}{ Yes } & No & $\boldsymbol{U}$ \\
Bone complication & $10.12(5.09)$ & $9.50(5.21)$ & 653.00 \\
Cardiac complication & $10.27(3.91)$ & $9.12(5.32)$ & 650.00 \\
Neurocognitive complication & $6.31(4.25)$ & $10.67(4.92)$ & $\mathbf{2 7 4 . 0 0 * *}$ \\
\hline
\end{tabular}

Legend: $* *=p<.01$. Significant results were highlighted in bold. 
Appendix Table 2. Mean levels of unmet needs according to morbidity status, and demographic and clinical characteristics $(\mathrm{n}=72)$.

\begin{tabular}{|c|c|c|c|c|c|c|c|c|c|c|c|c|}
\hline \multirow[b]{2}{*}{$\begin{array}{l}\text { Domains of unmet need } \\
(\text { mean - SD) }\end{array}$} & \multicolumn{3}{|c|}{ Bone } & \multicolumn{3}{|c|}{ Cardiac } & \multicolumn{3}{|c|}{ Neurocognitive } & \multicolumn{3}{|c|}{ Metabolic } \\
\hline & $\begin{array}{c}\text { Yes } \\
(\mathrm{n}=24)\end{array}$ & $\begin{array}{c}\text { No } \\
(n=48)\end{array}$ & $U$ & $\begin{array}{c}\text { Yes } \\
(\mathrm{n}=8)\end{array}$ & $\begin{array}{c}\text { No } \\
(n=64)\end{array}$ & $U$ & $\begin{array}{c}\text { Yes } \\
(\mathrm{n}=21)\end{array}$ & $\begin{array}{c}\text { No } \\
(\mathrm{n}=51)\end{array}$ & $\boldsymbol{U}$ & $\begin{array}{c}\text { Yes } \\
(\mathrm{n}=39)\end{array}$ & $\begin{array}{c}\text { No } \\
(n=33)\end{array}$ & $\boldsymbol{U}$ \\
\hline Information & $0.28(0.60)$ & $0.19(0.42)$ & 610.00 & $0.79(1.13)$ & $0.15(0.28)$ & 312.00 & $0.43(0.69)$ & $0.13(0.35)$ & $664.50 *$ & $0.28(0.61)$ & $0.15(0.28)$ & 663.50 \\
\hline Financial concerns & $0.48(0.69)$ & $0.42(0.54)$ & 580.00 & $0.69(0.91)$ & $0.41(0.54)$ & 282.50 & $0.70(0.82)$ & $0.34(0.45)$ & 648.00 & $0.47(0.71)$ & $0.41(0.42)$ & 659.50 \\
\hline Access and continuity of care & $0.48(0.65)$ & $0.18(0.52)$ & 738.50* & $0.69(1.11)$ & $0.23(0.47)$ & 315.50 & $0.42(0.63)$ & $0.23(0.56)$ & 637.50 & $0.30(0.62)$ & $0.27(0.53)$ & 647.50 \\
\hline Emotional health and relationship & $0.68(0.79)$ & $0.55(0.67)$ & 622.50 & $0.59(0.71)$ & $0.60(0.72)$ & 253.00 & $0.96(0.90)$ & $0.45(0.57)$ & $680.50 *$ & $0.70(0.78)$ & $0.48(0.61)$ & 723.50 \\
\hline
\end{tabular}

\begin{tabular}{|c|c|c|c|c|c|c|c|c|c|c|c|c|}
\hline \multirow[b]{2}{*}{$\begin{array}{l}\text { Domains of unmet need } \\
\text { (mean - SD) }\end{array}$} & \multicolumn{3}{|c|}{ Sex } & \multicolumn{3}{|c|}{ Age $^{\text {a }}$} & \multicolumn{3}{|c|}{ Marital status (single) } & \multicolumn{3}{|c|}{ Has children } \\
\hline & $\begin{array}{l}\text { Women } \\
(\mathrm{n}=34)\end{array}$ & $\begin{array}{c}\text { Men } \\
(n=38)\end{array}$ & $\boldsymbol{U}$ & $\begin{array}{c}<26 \\
\text { years } \\
(\mathrm{n}=37)\end{array}$ & $\begin{array}{c}>26 \\
\text { years } \\
(n=35)\end{array}$ & $\boldsymbol{U}$ & $\begin{array}{c}\text { Yes } \\
(n=38)\end{array}$ & $\begin{array}{c}\text { No } \\
(n=33)\end{array}$ & $\boldsymbol{U}$ & $\begin{array}{c}\text { Yes } \\
(n=19)\end{array}$ & $\begin{array}{c}\text { No } \\
(n=53)\end{array}$ & $\boldsymbol{U}$ \\
\hline Information & $0.20(0.38)$ & $0.21(0.56)$ & 677.00 & $0.29(0.57)$ & $0.14(0.36)$ & 538.50 & $0.27(0.57)$ & $0.14(0.36)$ & 710.50 & $0.19(0.45)$ & $0.23(0.50)$ & 465.00 \\
\hline Financial concerns & $0.36(0.60)$ & $0.52(0.59)$ & 489.50 & $0.56(0.61)$ & $0.31(0.56)$ & 441.00* & $0.53(0.61)$ & $0.35(0.57)$ & 763.00 & $0.39(0.70)$ & $0.46(0.56)$ & 491.50 \\
\hline Access and continuity of care & $0.31(0.51)$ & $0.26(0.64)$ & 707.50 & $0.33(0.67)$ & $0.23(0.48)$ & 579.50 & $0.32(0.66)$ & $0.22(0.48)$ & 697.00 & $0.28(0.55)$ & $0.28(0.60)$ & 428.50 \\
\hline Emotional health and relationship & $0.48(0.68)$ & $0.66(0.72)$ & 503.50 & $0.65(0.69)$ & $0.54(0.74)$ & 557.50 & $0.69(0.68)$ & $0.51(0.74)$ & 760.50 & $0.40(0.70)$ & $0.66(0.71)$ & 473.50 \\
\hline
\end{tabular}

\begin{tabular}{|c|c|c|c|c|c|c|c|c|c|c|c|c|}
\hline \multirow[b]{2}{*}{$\begin{array}{l}\text { Domains of unmet need } \\
\text { (mean - SD) }\end{array}$} & \multicolumn{3}{|c|}{$\begin{array}{c}\begin{array}{c}\text { Psychological follow-up } \\
\text { received }\end{array} \\
\end{array}$} & \multicolumn{3}{|c|}{ Time since diagnosis a } & \multicolumn{3}{|c|}{ ALL risk status (high) } & \multicolumn{3}{|c|}{ Radiotherapy received } \\
\hline & $\begin{array}{c}\text { Yes } \\
(n=18)\end{array}$ & $\begin{array}{c}\text { No } \\
(\mathrm{n}=54)\end{array}$ & $U$ & $\begin{array}{c}<16 \\
\text { years } \\
(\mathrm{n}=32)\end{array}$ & $\begin{array}{c}>16 \\
\text { years } \\
(n=40)\end{array}$ & $U$ & $\begin{array}{c}\text { Yes } \\
(n=52)\end{array}$ & $\begin{array}{l}\text { No } \\
(\mathrm{n}=19)\end{array}$ & $U$ & $\begin{array}{c}\text { Yes } \\
(n=58)\end{array}$ & $\begin{array}{c}\text { No } \\
(n=14)\end{array}$ & $U$ \\
\hline Information & $0.28(0.45)$ & $0.20(0.50)$ & 563.00 & $0.22(0.43)$ & $0.22(0.53)$ & 594.50 & $0.14(0.23)$ & $0.25(0.55)$ & 486.50 & $0.24(0.53)$ & $0.14(0.22)$ & 384.50 \\
\hline Financial concerns & $0.72(0.58)$ & $0.34(0.57)$ & $712.00 * *$ & $0.47(0.55)$ & $0.42(0.63)$ & 547.50 & $0.39(0.33)$ & $0.47(0.67)$ & 452.00 & $0.48(0.64)$ & $0.25(0.22)$ & 451.50 \\
\hline Access and continuity of care & $0.38(0.60)$ & $0.25(0.57)$ & 583.50 & $0.34(0.71)$ & $0.23(0.45)$ & 625.50 & $0.34(0.53)$ & $0.27(0.60)$ & 347.00* & $0.26(0.58)$ & $0.37(0.59)$ & 310.00 \\
\hline Emotional health and relationship & $0.92(0.86)$ & $0.49(0.62)$ & $635.00 *$ & $0.53(0.65)$ & $0.64(0.76)$ & 690.00 & $0.67(0.59)$ & $0.58(0.76)$ & 406.50 & $0.61(0.75)$ & $0.52(0.53)$ & 403.50 \\
\hline
\end{tabular}

Legend: ${ }^{a}=$ groups were created to be as homogeneous as possible; $\mathrm{ALL}=$ acute lymphoblastic leukemia; $\mathrm{SD}=$ standard deviation; $*=p<.05 ; * *=p<.01$. Significant results were highlighted in bold 
Appendix Table 3. Mean levels of unmet needs according to the presence of metabolic $(\mathrm{n}=15)$, bone $(\mathrm{n}=10)$ or neurocognitive $(\mathrm{n}=5)$ morbidity alone (comorbidity excluded).

\begin{tabular}{lcccc}
\hline & \multicolumn{3}{c}{ Late adverse effect } \\
\cline { 2 - 5 } $\begin{array}{l}\text { Domains of unmet need } \\
\text { (mean - SD) }\end{array}$ & $\begin{array}{c}\text { Metabolic } \\
(\mathrm{n}=15)\end{array}$ & $\begin{array}{c}\text { Bourocognitive } \\
(\mathrm{n}=10)\end{array}$ & $\begin{array}{c}\text { Kruska } \\
\text { l-Wallis }\end{array}$ \\
\hline Information & $0.04(0.17)$ & $0.30(0.40)$ & $0.20(0.30)$ & $\boldsymbol{H}$ \\
Financial concerns & $0.23(0.29)$ & $0.38(0.52)$ & $0.50(0.66)$ & 4.50 \\
Access and continuity of care & $0.11(0.26)$ & $0.35(0.58)$ & $0.33(0.58)$ & 1.70 \\
Emotional health and relationship & $0.40(0.44)$ & $0.69(0.78)$ & $0.57(0.66)$ & 1.04 \\
\hline
\end{tabular}

Legend: $\mathrm{SD}=$ standard deviation.

Appendix Table 4. Association between unmet needs, pain and functional status.

\begin{tabular}{llll}
\hline Measures & $\begin{array}{c}\text { BPI pain severity } \\
(\mathrm{n}=68)\end{array}$ & $\begin{array}{c}\text { BPI pain } \\
\text { interference } \\
(\mathrm{n}=68)\end{array}$ & $\begin{array}{c}\text { Functional status } \\
\text { 15D } \\
(\mathrm{n}=66)\end{array}$ \\
\hline $\begin{array}{l}\text { Domains of unmet needs } \\
\text { Information }\end{array}$ & 0.22 & $0.41^{*}$ & $-0.32^{*}$ \\
$\quad$ Financial concerns & 0.17 & 0.24 & $-0.25^{*}$ \\
Access and continuity of care & $0.30^{*}$ & $0.43^{* *}$ & $-0.34^{* *}$ \\
$\quad$ Emotional health and relationship & $0.24^{*}$ & $0.37^{* *}$ & $-0.47^{* *}$ \\
\hline Mean (SD) & $1.71(1.85)$ & $1.17(1.78)$ & $0.92(0.08)$ \\
Scope & $0-6,5$ & $0-7,29$ & $0.68-1$ \\
\hline
\end{tabular}

Legend: $15 \mathrm{D}=$ instrument of health-related quality of life; $\mathrm{BPI}=$ brief pain inventory; $\mathrm{SD}=$ standard deviation; $*=p<.05$; $* *=p<.01$. 